\title{
GODUNOV METHOD FOR NONCONSERVATIVE HYPERBOLIC SYSTEMS
}

\author{
María Luz Muñoz-Ruiz ${ }^{1}$ and CARlos ParÉs ${ }^{2}$
}

\begin{abstract}
This paper is concerned with the numerical approximation of Cauchy problems for onedimensional nonconservative hyperbolic systems. The theory developed by Dal Maso et al. [J. Math. Pures Appl. 74 (1995) 483-548] is used in order to define the weak solutions of the system: an interpretation of the nonconservative products as Borel measures is given, based on the choice of a family of paths drawn in the phase space. Even if the family of paths can be chosen arbitrarily, it is natural to require this family to satisfy some hypotheses concerning the relation of the paths with the integral curves of the characteristic fields. The first goal of this paper is to investigate the implications of three basic hypotheses of this nature. Next, we show that, when the family of paths satisfies these hypotheses, Godunov methods can be written in a natural form that generalizes their classical expression for systems of conservation laws. We also study the well-balance properties of these methods. Finally, we prove the consistency of the numerical scheme with the definition of weak solutions: we prove that, under hypothesis of bounded total variation, if the approximations provided by a Godunov method based on a family of paths converge uniformly to some function as the mesh is refined, then this function is a weak solution (related to that family of paths) of the nonconservative system. We extend this result to a family of numerical schemes based on approximate Riemann solvers.
\end{abstract}

Mathematics Subject Classification. 74S10, 35L60, 65M12.

Received: May 21, 2006. Revised: January 5, 2006.

\section{INTRODUCTION}

This paper is concerned with the numerical approximation of Cauchy problems for one-dimensional nonconservative hyperbolic systems:

$$
W_{t}+\mathcal{A}(W) W_{x}=0, \quad x \in \mathbb{R}, t>0 .
$$

We will assume that the system is strictly hyperbolic and that the characteristic fields are either genuinely nonlinear or linearly degenerate.

A particular class of PDE systems that can be written in the form (1.1) is given by

$$
W_{t}+F(W)_{x}=B(W) W_{x}+S(W) \sigma_{x},
$$

Keywords and phrases. Nonconservative hyperbolic systems, Godunov method, well-balancing, approximate Riemann solvers.

1 Dept. Matemática Aplicada, Universidad de Málaga, 29071-Málaga, Spain.

2 Dept. Análisis Matemático, Universidad de Málaga, 29071-Málaga, Spain. 
where the unknown $W(x, t)$ takes values on an open convex set $\Omega$ of $\mathbb{R}^{N} ; F$ is a regular function from $\Omega$ to $\mathbb{R}^{N}$; $B$ is a regular matrix function from $\Omega$ to $\mathcal{M}_{N}(\mathbb{R}) ; S$ a function from $\Omega$ to $\mathbb{R}^{N}$; and $\sigma(x)$ is a known function from $\mathbb{R}$ to $\mathbb{R}$. In effect, adding to (1.2) the trivial equation

$$
\sigma_{t}=0,
$$

this system can be easily rewritten in the form of a quasilinear first order system as (1.1) (see $[8-11,16]$ ). System (1.2) includes as a particular case systems of conservation laws $(B=0, S=0)$, systems of conservation laws with source term or balance laws $(B=0)$ and coupled systems of conservation laws as defined in [3]. Such systems occur in many applications, as multiphase flows or multilayer fluid models.

A first difficulty related with systems (1.1) comes from the presence of nonconservative products, which makes difficult the definition of weak solutions: in general, the nonconservative product $\mathcal{A}(W) W_{x}$ does not make sense as a distribution. Dal Maso et al. proposed in [5] an interpretation of these products as Borel measures, based on the choice of a family of paths in the phase space: a recent exposition on this subject can be found in [17]. We briefly recall this theory in Section 2.

Together with the definition of weak solutions, a notion of entropy has to be chosen, as the usual Lax's concept or one related to an entropy pair. Once this choice is done, the theory of simple waves of hyperbolic systems of conservation laws and the results concerning the solutions of Riemann problems can be extended to systems of the form (1.1).

The choice of the family of paths is important because it determines the speed of propagation of shocks. The simplest choice is given by the family of segments, that corresponds to the definition of nonconservative products proposed by Volpert [22]. In practical applications, it has to be based on the physical background of the problem. In [16] a clear motivation for the selection of the family of paths is provided when a physical regularization by diffusion, dispersion, etc., is available.

Even if the family of paths can be chosen arbitrarily, it is natural from the mathematical point of view to require this family to satisfy some hypotheses concerning the relation of the paths with the integral curves of the characteristic fields. The first goal of this paper is to investigate the implications of three basic hypotheses of this nature. This is done in Section 3 where we show that, when these hypotheses are satisfied, there is a strong relation between the path linking two close states and the total mass of the Borel measure associated to the solution of the Riemann problem having these states as initial condition. A family of paths satisfying these hypotheses can always be constructed at least for states that are near enough in a sense to be determined.

In this article we are concerned with Godunov methods for systems of the form (1.1). As it is well known, these methods give approximations of the solution of Cauchy problems by means of the exact resolution of Riemann problems at the intercells. In Section 4 we show that, when the family of paths satisfies the above mentioned hypotheses, these methods can be written in a natural form that generalizes the classical expression of the Godunov method for systems of conservation laws. We also study the well-balance properties of these methods. Well-balancing is related to the numerical approximation of equilibria, i.e., steady solutions (see $[1,8-11,19-21])$.

A basic requirement for a numerical method for (1.1) is the following: if the sequence of numerical solutions converges as the grid becomes infinitely fine, the limit must be a weak solution. In the particular case of systems of conservation laws, the Lax-Wendroff convergence theorem [15] states that conservative numerical schemes have this property. In Section 5 we prove a Lax-Wendroff type convergence theorem for the Godunov method presented in the previous section: if the sequence of approximations obtained with a Godunov method based on the family of paths used to define the concept of weak solutions (satisfying the basic hypotheses) has bounded total variation and it converges uniformly as the mesh size tends to 0 , its limit is a weak solution of system (1.1).

Godunov methods as described here are particular cases of the class of path-conservative numerical schemes introduced in [20]. In the final section we consider more general path-conservative numerical schemes based on approximate Riemann solvers and we give a sufficient condition that makes possible to extend the consistency result shown for Godunov methods. 


\section{WEAK SOLUTiONS}

Consider the problem in nonconservative form

$$
W_{t}+\mathcal{A}(W) W_{x}=0, \quad x \in \mathbb{R}, t>0,
$$

where $W(x, t)$ belongs to $\Omega$, an open convex subset of $\mathbb{R}^{N}$, and $W \in \Omega \mapsto \mathcal{A}(W) \in \mathcal{M}_{N}(\mathbb{R})$ is a smooth locally bounded map. We suppose that system (2.1) is strictly hyperbolic, that is, for each $W \in \Omega$ the matrix $\mathcal{A}(W)$ has $N$ real distinct eigenvalues $\lambda_{1}(W)<\cdots<\lambda_{N}(W)$, with associated eigenvectors $R_{1}(W), \ldots, R_{N}(W)$. We also suppose that for each $i=1, \ldots, N$, the characteristic field $R_{i}(W)$ is either genuinely nonlinear:

$$
\nabla \lambda_{i}(W) \cdot R_{i}(W) \neq 0, \quad \forall W \in \Omega,
$$

or linearly degenerate:

$$
\nabla \lambda_{i}(W) \cdot R_{i}(W)=0, \quad \forall W \in \Omega .
$$

In general, the nonconservative product $\mathcal{A}(W) W_{x}$ does not make sense as a distribution. Nevertheless, after the theory developed by Dal Maso et al. [5] it is possible to give a rigorous definition of weak solutions associated to the choice of a family of paths in $\Omega$ :

Definition 2.1. A family of paths in $\Omega \subset \mathbb{R}^{N}$ is a locally Lipschitz map

$$
\Phi:[0,1] \times \Omega \times \Omega \rightarrow \Omega
$$

such that:

- $\Phi\left(0 ; W_{L}, W_{R}\right)=W_{L}$ and $\Phi\left(1 ; W_{L}, W_{R}\right)=W_{R}$, for any $W_{L}, W_{R} \in \Omega$.

- For every arbitrary bounded set $\mathcal{O} \subset \Omega$, there exists a constant $k$ such that

$$
\left|\frac{\partial \Phi}{\partial s}\left(s ; W_{L}, W_{R}\right)\right| \leq k\left|W_{L}-W_{R}\right|,
$$

for any $W_{L}, W_{R} \in \mathcal{O}$ and almost every $s \in[0,1]$.

- For every bounded set $\mathcal{O} \subset \Omega$, there exists a constant $K$ such that

$$
\left|\frac{\partial \Phi}{\partial s}\left(s ; W_{L}^{1}, W_{R}^{1}\right)-\frac{\partial \Phi}{\partial s}\left(s ; W_{L}^{2}, W_{R}^{2}\right)\right| \leq K\left(\left|W_{L}^{1}-W_{L}^{2}\right|+\left|W_{R}^{1}-W_{R}^{2}\right|\right),
$$

for any $W_{L}^{1}, W_{R}^{1}, W_{L}^{2}, W_{R}^{2} \in \mathcal{O}$ and almost every $s \in[0,1]$.

Once a family of paths $\Phi$ has been chosen, the nonconservative product $\mathcal{A}(W) W_{x}$ can be interpreted as a Borel measure for $W \in\left(L^{\infty}\left(\mathbb{R} \times \mathbb{R}^{+}\right) \cap B V\left(\mathbb{R} \times \mathbb{R}^{+}\right)\right)^{N}$, denoted by $\left[\mathcal{A}(W) W_{x}\right]_{\Phi}$. Given a time $t$, the Borel measure related to the nonconservative product is defined as follows:

$$
\begin{aligned}
\left\langle\left[\mathcal{A}(W(\cdot, t)) W_{x}(\cdot, t)\right]_{\Phi}, \varphi\right\rangle= & \int_{\mathbb{R}} \mathcal{A}(W(x, t)) W_{x}(x, t) \varphi(x) \mathrm{d} x \\
& +\sum_{m}\left(\int_{0}^{1} \mathcal{A}\left(\Phi\left(s ; W_{m}^{-}, W_{m}^{+}\right)\right) \frac{\partial \Phi}{\partial s}\left(s ; W_{m}^{-}, W_{m}^{+}\right) \mathrm{d} s\right) \varphi\left(x_{m}(t)\right), \quad \forall \varphi \in \mathcal{C}_{0}(\mathbb{R}) .
\end{aligned}
$$

In the above equality, $x_{m}(t)$ are the locations of the discontinuities of $W$ at time $t$, which are countable; $W_{m}^{-}$and $W_{m}^{+}$are respectively the limits of $W$ to the left and to the right at the $m$-th discontinuity at time $t$; and $\mathcal{C}_{0}(\mathbb{R})$ is the set of continuous maps with compact support. 
Definition 2.2. A function $W \in\left(L^{\infty}\left(\mathbb{R} \times \mathbb{R}^{+}\right) \cap B V\left(\mathbb{R} \times \mathbb{R}^{+}\right)\right)^{N}$ which is piecewise $\mathcal{C}^{1}$ is said to be a weak solution of (2.1) if it satisfies the equality

$$
W_{t}+\left[\mathcal{A}(W) W_{x}\right]_{\Phi}=0 .
$$

When no confusion arises, the dependency on $\Phi$ will be dropped.

Across a discontinuity, a weak solution must satisfy the generalized Rankine-Hugoniot condition:

$$
\int_{0}^{1}\left(\sigma \mathcal{I}-\mathcal{A}\left(\Phi\left(s ; W^{-}, W^{+}\right)\right)\right) \frac{\partial \Phi}{\partial s}\left(s ; W^{-}, W^{+}\right) \mathrm{d} s=0,
$$

where $\sigma$ is the speed of propagation of the discontinuity, $\mathcal{I}$ is the identity matrix, and $W^{-}$and $W^{+}$are the left and right limits of the solution at the discontinuity.

In the particular case of a system of conservation laws, that is, when $\mathcal{A}(W)$ is the Jacobian matrix of some flux function $F(W)$, the definition of the nonconservative product as a Borel measure does not depend on the choice of paths, and the generalized Rankine-Hugoniot condition reduces to the usual one.

As it occurs in the conservative case, not every discontinuity is admissible. Therefore, a concept of entropy solution has to be assumed, as one of the following ones:

Definition 2.3. A weak solution is said to be an entropy solution in the Lax sense if, at each discontinuity, there exists $i \in\{1, \ldots, N\}$ such that

$$
\lambda_{i-1}\left(W^{-}\right)<\sigma<\lambda_{i}\left(W^{-}\right) \quad \text { and } \quad \lambda_{i}\left(W^{+}\right)<\sigma<\lambda_{i+1}\left(W^{+}\right)
$$

if the $i$-th characteristic field is genuinely nonlinear or

$$
\lambda_{i}\left(W^{-}\right)=\sigma=\lambda_{i}\left(W^{+}\right)
$$

if the $i$-th characteristic field is linearly degenerate.

Definition 2.4. Given an entropy pair $(\eta, G)$ for $(2.1)$, i.e. a pair of regular functions from $\Omega$ to $\mathbb{R}$ such that

$$
\nabla G(W)=\nabla \eta(W) \cdot \mathcal{A}(W), \quad \forall W \in \Omega,
$$

a weak solution is said to be entropy satisfying if the inequality

$$
\eta(W)_{t}+G(W)_{x} \leq 0
$$

is satisfied in the distributions sense.

\section{Choice of PAThS}

In the definition of weak solutions of system (2.1), the choice of the family of paths $\Phi$ is important because it determines the speed of shocks. In this article, we shall assume that the family of paths satisfies the following hypotheses:

(H1) Given two states $W_{L}$ and $W_{R}$ belonging to the same integral curve $\gamma$ of a linearly degenerate field, the path $\Phi\left(\cdot ; W_{L}, W_{R}\right)$ is a parametrization of the arc of $\gamma$ linking $W_{L}$ and $W_{R}$.

(H2) Given two states $W_{L}$ and $W_{R}$ belonging to the same integral curve $\gamma$ of a genuinely nonlinear field, $R_{i}$, and such that $\lambda_{i}\left(W_{L}\right)<\lambda_{i}\left(W_{R}\right)$, the path $\Phi\left(\cdot ; W_{L}, W_{R}\right)$ is a parametrization of the arc of $\gamma$ linking $W_{L}$ and $W_{R}$. 
(H3) Let us denote by $\mathcal{R} \mathcal{P} \subset \Omega \times \Omega$ the set of pairs $\left(W_{L}, W_{R}\right)$ such that the Riemann problem

$$
\left\{\begin{array}{l}
W_{t}+\mathcal{A}(W) W_{x}=0 \\
W(x, 0)= \begin{cases}W_{L} & \text { if } x<0 \\
W_{R} & \text { if } x>0\end{cases}
\end{array}\right.
$$

has a unique self-similar weak solution composed by at most $N$ simple waves (i.e. entropy shocks, contact discontinuities or rarefaction waves) connecting $J+1$ intermediate constant states

$$
W_{0}=W_{L}, W_{1}, \ldots, W_{J-1}, W_{J}=W_{R}
$$

with $J \leq N$. Then, given $\left(W_{L}, W_{R}\right) \in \mathcal{R} \mathcal{P}$, the curve described by the path $\Phi\left(\cdot ; W_{L}, W_{R}\right)$ in $\Omega$ is equal to the union of those corresponding to the paths $\Phi\left(\cdot ; W_{j-1}, W_{j}\right), j=1, \ldots, J$.

The reason to set these hypotheses is that they allow us to prove the three following natural properties:

Proposition 3.1. Let us assume that the concept of weak solutions of (2.1) is defined on the basis of a family of paths satisfying hypotheses (H1)-(H3). Then:

(i) Given two states $W_{L}$ and $W_{R}$ belonging to the same integral curve of a linearly degenerate field, the contact discontinuity given by

$$
W(x, t)= \begin{cases}W_{L} & \text { if } x<\sigma t, \\ W_{R} & \text { if } x>\sigma t,\end{cases}
$$

where $\sigma$ is the (constant) value of the corresponding eigenvalue through the integral curve, is an entropy weak solution of (2.1).

(ii) Let $\left(W_{L}, W_{R}\right)$ be a pair belonging to $\mathcal{R P}$ and let $W$ be the solution of the Riemann problem (3.1). The following equality holds for every $t>0$ :

$$
\left\langle\mathcal{A}(W(\cdot, t)) W_{x}(\cdot, t), 1\right\rangle=\int_{0}^{1} \mathcal{A}\left(\Phi\left(s ; W_{L}, W_{R}\right)\right) \frac{\partial \Phi}{\partial s}\left(s ; W_{L}, W_{R}\right) \mathrm{d} s .
$$

Consequently, the total mass of the Borel measure $\mathcal{A}(W(\cdot, t)) W_{x}(\cdot, t)$ does not depend on $t$.

(iii) Let $\left(W_{L}, W_{R}\right)$ be a pair belonging to $\mathcal{R P}$ and $W_{j}$ any of the intermediate states involved by the solution of the Riemann problem (3.1). Then:

$$
\begin{aligned}
\int_{0}^{1} \mathcal{A}\left(\Phi\left(s ; W_{L}, W_{R}\right)\right) \frac{\partial \Phi}{\partial s}\left(s ; W_{L}, W_{R}\right) \mathrm{d} s=\int_{0}^{1} \mathcal{A}\left(\Phi\left(s ; W_{L}, W_{j}\right)\right) \frac{\partial \Phi}{\partial s}\left(s ; W_{L}, W_{j}\right) \mathrm{d} s & \\
& +\int_{0}^{1} \mathcal{A}\left(\Phi\left(s ; W_{j}, W_{R}\right)\right) \frac{\partial \Phi}{\partial s}\left(s ; W_{j}, W_{R}\right) \mathrm{d} s .
\end{aligned}
$$

Proof. The proof of (i) is straightforward.

To prove (ii), let us denote by $W_{0}=W_{L}, W_{1}, \ldots, W_{J-1}, W_{J}=W_{R}$, with $J \leq N$, the intermediate states involved by the solution of (3.1).

First, notice that in regions where $W(\cdot, t)$ is constant, the corresponding mass is zero, as the derivative $W_{x}(\cdot, t)$ vanishes. Taking this fact into account in $(2.2)$, the total mass can be written in this particular case as follows:

$$
\left\langle\mathcal{A}(W(\cdot, t)) W_{x}(\cdot, t), 1\right\rangle=\sum_{j \in J_{1}} \int_{\sigma_{j}^{-} t}^{\sigma_{j}^{+} t} \mathcal{A}(W(x, t)) W_{x}(x, t) \mathrm{d} x+\sum_{j \in J_{2}} \int_{0}^{1} \mathcal{A}\left(\Phi\left(s ; W_{j-1}, W_{j}\right)\right) \frac{\partial \Phi}{\partial s}\left(s ; W_{j-1}, W_{j}\right) \mathrm{d} s,
$$


where $\left\{J_{1}, J_{2}\right\}$ is a partition of $\{1, \ldots, J\}$ defined as follows: $j \in J_{1}$ if $W_{j-1}$ and $W_{j}$ are linked by a rarefaction wave; $j \in J_{2}$ if $W_{j-1}$ and $W_{j}$ are linked by a shock or a contact discontinuity. Given $j \in J_{1}$, we denote by $\sigma_{j}^{-}$ and $\sigma_{j}^{+}$the speeds of the left and right front of the corresponding wave.

Notice that if $j \in J_{1}$ then $W_{j-1}$ and $W_{j}$ lie on the same integral curve of a genuinely nonlinear characteristic field. Moreover, given $t>0$ the map $x \in\left[\sigma_{j}^{-} t, \sigma_{j}^{+} t\right] \mapsto W(x, t) \in \Omega$ can be interpreted as a parametrization of the arc of the integral curve linking the states (see [7]). Therefore, taking into account (H2), after a reparametrization we have:

$$
\int_{\sigma_{j}^{-} t}^{\sigma_{j}^{+} t} \mathcal{A}(W(x, t)) W_{x}(x, t) \mathrm{d} x=\int_{0}^{1} \mathcal{A}\left(\Phi\left(s ; W_{j-1}, W_{j}\right)\right) \frac{\partial \Phi}{\partial s}\left(s ; W_{j-1}, W_{j}\right) \mathrm{d} s .
$$

As a consequence, (3.2) can be rewritten as follows:

$$
\left\langle\mathcal{A}(W(\cdot, t)) W_{x}(\cdot, t), 1\right\rangle=\sum_{j=1}^{J}\left(\int_{0}^{1} \mathcal{A}\left(\Phi\left(s ; W_{j-1}, W_{j}\right)\right) \frac{\partial \Phi}{\partial s}\left(s ; W_{j-1}, W_{j}\right) \mathrm{d} s\right) .
$$

Property (ii) is easily deduced from the latter equality and hypothesis (H3).

Finally, property (iii) is a straighforward consequence of (ii).

Remark 3.2. Hypothesis (H1) for linearly degenerate fields is very classical and it has been assumed by many authors (see for instance $[4,9,14]$ ). The importance of the assumption (H3) has been pointed out in [17]. Nevertheless (H2) has not been considered previously, to our knowledge. Even if, unlike (H1), hypotheses (H2) and (H3) do not give any information about the speed of propagation of discontinuities, they allow to prove the relations between the paths and the solutions of Riemann problems given by (ii) and (iii). These relations make easier the extension to nonconservative problems of classical families of numerical schemes for systems of conservations laws, as Godunov methods or methods based on approximate Riemann solvers, as it will be shown hereafter.

A general procedure to construct a family of paths satistying $(\mathrm{H} 1)-(\mathrm{H} 3)$, at least for the class $\mathcal{R} \mathcal{P}$, is as follows:

1. For each genuinely nonlinear field $R_{i}$ we choose a family of paths $\Phi_{i}$. The Rankine-Hugoniot conditions for shocks associated to that field will involve this family of paths:

$$
\int_{0}^{1}\left(\sigma \mathcal{I}-\mathcal{A}\left(\Phi_{i}\left(s ; W_{L}, W_{R}\right)\right)\right) \frac{\partial \Phi_{i}}{\partial s}\left(s ; W_{L}, W_{R}\right) \mathrm{d} s=0 .
$$

In general, the choice of these families has to be based on the physical background of the problem, and it can be different for every genuinely nonlinear field.

2. (H1) and (H2) are used to define the path linking two states belonging to a same integral curve of a characteristic field.

3. Using only the paths defined above, it is possible to extend the theory of simple waves of hyperbolic systems of conservation laws to hyperbolic nonconservative systems (2.1) (see [5]): for each $i=1, \ldots, N$ and $W_{L} \in \Omega$ it is possible to construct a curve $\varepsilon \mapsto \omega_{i}\left(\varepsilon ; W_{L}\right) \in \Omega$, for $|\varepsilon|$ small enough, such that:

- If the $i$-th characteristic field is genuinely nonlinear, the states $W_{L}$ and $W_{R}=\omega_{i}\left(\varepsilon ; W_{L}\right)$ can be connected by an $i$-shock wave satisfying (3.3) for $\varepsilon<0$, and by an $i$-rarefaction wave for $\varepsilon>0$.

- If the $i$-th characteristic field is linearly degenerate, $W_{L}$ and $W_{R}=\omega_{i}\left(\varepsilon ; W_{L}\right)$ can be connected by an $i$-contact discontinuity for every $\varepsilon$.

4. Now it is possible to solve the Riemann problem (3.1) when the states $W_{L}$ and $W_{R}$ are sufficiently close. The following result can be proved (see $[5,18])$ :

Theorem 3.3. For all $W_{L} \in \Omega$ there exists a neighborhood $\mathcal{O}$ of $W_{L}$ in $\Omega$ such that, if $W_{R}$ belongs to $\mathcal{O}$, the Riemann problem (3.1) has a unique weak solution that consists of $J+1$ constant states 
$W_{0}=W_{L}, W_{1}, \ldots, W_{J-1}, W_{J}=W_{R}, J \leq N$, connected by rarefaction waves, entropy shock waves, or contact discontinuities.

5. Hypothesis (H3) is finally used to define the path linking a pair of states belonging to $\mathcal{R} \mathcal{P}$.

Observe that, if $W_{L}$ and $W_{R}$ are connected by an $i$-shock, conditions (2.3) and (3.3) coincide.

Remark 3.4. In some cases the mathematical aspects of the problem can give enough information to select the family of paths $\Phi_{i}$ for some of the genuinely nonlinear fields: let us suppose, for instance, that there exists a regular function $F: \Omega \rightarrow \mathbb{R}^{K}$, with $K \leq N$, and two sets of indexes $\left\{i_{1}, \ldots, i_{K}\right\}$ and $\left\{j_{1}, \ldots, j_{K}\right\}$ such that

$$
\frac{\partial F_{i_{p}}}{\partial w_{j_{q}}}(W)=a_{i_{p}, j_{q}}(W), \quad \forall p, q \in\{1, \ldots, K\}, \forall W \in \Omega,
$$

and

$$
a_{i, j_{k}}(W)=0, \quad \forall i \notin\left\{i_{1}, \ldots, i_{K}\right\}, k \in\{1, \ldots, K\},
$$

where $a_{i, j}(W)$ denotes the $(i, j)$ entry of the matrix $\mathcal{A}(W)$. Moreover, let us suppose that the $K \times K$ matrix formed from the entries of $\mathcal{A}(W)$ by selecting only the rows $i_{1}, \ldots, i_{k}$ and the columns $j_{1}, \ldots, j_{k}$ has $K$ different real eigenvalues $\lambda_{1}(W), \ldots, \lambda_{K}(W)$, with associated eigenvectors $r_{1}(W), \ldots, r_{K}(W)$. We can assume without loss of generality that $i_{k}=j_{k}=k, 1 \leq k \leq K$. The structure of the matrix is then as follows:

$$
\mathcal{A}(W)=\left[\begin{array}{cc}
A_{1,1}(W) & A_{1,2}(W) \\
0 & A_{2,2}(W)
\end{array}\right]
$$

being $A_{1,1}(W)$ the Jacobian of $F$ with respect to the first $K$ variables. Let us consider the following family of systems of conservation laws:

$$
\frac{\partial}{\partial t}\left[\begin{array}{c}
w_{1} \\
\vdots \\
w_{K}
\end{array}\right]+\frac{\partial F}{\partial x}\left(w_{1}, \ldots, w_{K}, \bar{w}_{K+1}, \ldots, \bar{w}_{N}\right)=0
$$

where $\bar{w}_{j}, j=K+1, \ldots, N$, are fixed constant values. It is trivial to show that

$$
\left[w_{1}(x, t), \ldots, w_{K}(x, t)\right]^{T}
$$

is a classical solution of (3.4) if and only if

$$
\left[w_{1}(x, t), \ldots, w_{K}(x, t), \bar{w}_{K+1}, \ldots, \bar{w}_{N}\right]^{T}
$$

is a classical solution of (2.1). Therefore, it is natural to define the weak solutions in order to preserve this relation. Notice that, in this case, the first $K$ characteristic fields of (2.1) are given by

$$
R_{i}(W)=\left[\begin{array}{c}
r_{i}(W) \\
0
\end{array}\right]
$$

Let us assume that the corresponding families of paths $\Phi_{i}$ are chosen so that, given two states

$$
W_{L}=\left[\begin{array}{c}
w_{1}^{L} \\
\vdots \\
w_{N}^{L}
\end{array}\right], \quad W_{R}=\left[\begin{array}{c}
w_{1}^{R} \\
\vdots \\
w_{N}^{R}
\end{array}\right]
$$

such that

$$
w_{j}^{L}=w_{j}^{R}=\bar{w}_{j}, \quad j=K+1, \ldots, N,
$$


then

$$
\left(\Phi_{i}\right)_{j}\left(s ; W_{L}, W_{R}\right)=\bar{w}_{j}, \quad j=K+1, \ldots, N
$$

where $\left(\Phi_{i}\right)_{j}, j=1, \ldots, N$, represent the components of $\Phi_{i}$. Then, it can be easily shown that the jump condition (3.3) does not depend on the particular choice of $\Phi_{i}$ and it reduces to the usual Rankine-Hugoniot condition:

$$
F\left(W_{R}\right)-F\left(W_{L}\right)=\sigma\left(W_{R}-W_{L}\right)
$$

As a consequence, $\left[w_{1}(x, t), \ldots, w_{K}(x, t)\right]^{T}$ is a weak solution of $(3.4)$ if and only if $\left[w_{1}(x, t), \ldots, w_{K}(x, t)\right.$, $\left.\bar{w}_{K+1}, \ldots, \bar{w}_{N}\right]^{T}$ is a weak solution of $(2.1)$. In this case, any family of paths $\Phi_{i}$ satisfying the above hypothesis can be selected. In particular, $\Phi_{i}$ can be chosen as the family of segments:

$$
\Phi_{i}\left(s ; W_{L}, W_{R}\right)=W_{L}+s\left(W_{R}-W_{L}\right) .
$$

Remark 3.5. A system of conservation laws with source term

$$
W_{t}+F(W)_{x}=S(W) \sigma_{x},
$$

can be considered as a particular case of (2.1) if the trivial equation

$$
\sigma_{t}=0
$$

is added to the system. Then, the system can be formulated as follows:

$$
\widetilde{W}_{t}+\widetilde{\mathcal{A}}(\widetilde{W}) W_{x}=0
$$

where $\widetilde{W}$ is the augmented vector

$$
\widetilde{W}=\left[\begin{array}{l}
W \\
\sigma
\end{array}\right]
$$

and the block structure of $\widetilde{\mathcal{A}}(\widetilde{W})$ is given by

$$
\widetilde{\mathcal{A}}(\widetilde{W})=\left[\begin{array}{cc}
A(W) & -S(W) \\
0 & 0
\end{array}\right],
$$

being $A(W)$ the Jacobian matrix of the flux function $F$. Let us suppose that, for any $W$, the matrix $A(W)$ has $N$ real distinct eigenvalues

with associated eigenvectors

$$
\lambda_{1}(W), \ldots, \lambda_{N}(W),
$$

$$
R_{1}(W), \ldots, R_{N}(W)
$$

and the characteristic fields are either genuinely nonlinear or linearly degenerate. While $\lambda_{i}(W) \neq 0, i=1, \ldots, N$, system (3.7) is strictly hyperbolic: the eigenvalues of the matrix are

$$
\lambda_{1}(W), \ldots, \lambda_{N}(W), \lambda_{*}(W)=0,
$$

with associated eigenvectors

$$
\widetilde{R}_{i}(\widetilde{W})=\left[\begin{array}{c}
R_{i}(W) \\
0
\end{array}\right], i=1, \ldots, N ; \quad \widetilde{R}_{*}(\widetilde{W})=\left[\begin{array}{c}
A^{-1}(W) S(W) \\
1
\end{array}\right] .
$$

Moreover, for $i=1, \ldots, N$, the characteristic field $\widetilde{R}_{i}$ has the same character of $R_{i}$, and the $(N+1)$-th characteristic field is linearly degenerate. Notice that system (3.7) fulfills the hypotheses stated in Remark 3.4 with $K=N$. According to this remark and following the guidelines given above, a family of paths satisfying (H1)-(H3) is completely determined at least for pairs $\left(W_{L}, W_{R}\right) \in \mathcal{R} \mathcal{P}$. 


\section{Godunov method FOR NONCONSERVATIVE HYPERBOliC SYSTEMS}

We consider system (2.1) in the conditions stated in Section 2, with initial condition

$$
W(x, 0)=W_{0}(x), \quad x \in \mathbb{R} .
$$

We assume that the family of paths $\Phi$ used in the definition of the nonconservative product $\mathcal{A}(W) W_{x}$ satisfies the hypotheses (H1)-(H3).

For the discretization of the system, computing cells $I_{i}=\left[x_{i-1 / 2}, x_{i+1 / 2}\right]$ are considered. For simplicity, we suppose that these cells have constant size $\Delta x$ and that $x_{i+\frac{1}{2}}=i \Delta x$. Define $x_{i}=(i-1 / 2) \Delta x$, the center of the cell $I_{i}$. Let $\Delta t$ be the constant time step and define $t^{n}=n \Delta t$.

We denote by $W_{i}^{n}$ the approximation of the cell averages of the exact solution provided by the numerical scheme:

$$
W_{i}^{n} \cong \frac{1}{\Delta x} \int_{x_{i-1 / 2}}^{x_{i+1 / 2}} W\left(x, t^{n}\right) \mathrm{d} x
$$

Suppose that the averages $W_{i}^{n}$ at time $t=t^{n}$ are known. As it is usual in Godunov-type methods, to advance in time we consider the Riemann problems

$$
\left\{\begin{array}{l}
W_{t}^{i+1 / 2}+\mathcal{A}\left(W^{i+1 / 2}\right) W_{x}^{i+1 / 2}=0 \\
W^{i+1 / 2}\left(x, t^{n}\right)= \begin{cases}W_{i}^{n} & \text { if } x<x_{i+1 / 2} \\
W_{i+1}^{n} & \text { if } x>x_{i+1 / 2}\end{cases}
\end{array}\right.
$$

and we approximate the solution at time $t^{n+1}$ by

$$
W_{i}^{n+1}=\frac{1}{\Delta x}\left(\int_{x_{i-1 / 2}}^{x_{i}} W^{i-1 / 2}\left(x, t^{n+1}\right) \mathrm{d} x+\int_{x_{i}}^{x_{i+1 / 2}} W^{i+1 / 2}\left(x, t^{n+1}\right) \mathrm{d} x\right) .
$$

If a CFL condition $1 / 2$ is assumed, calculating for every $t$ the Borel measure $W_{t}^{i+1 / 2}+\mathcal{A}\left(W^{i+1 / 2}\right) W_{x}^{i+1 / 2}$ of $\left[x_{i}, x_{i+1 / 2}\right]$ and integrating in $\left[t^{n}, t^{n+1}\right]$ we obtain:

$$
\int_{x_{i}}^{x_{i+1 / 2}} W^{i+1 / 2}\left(x, t^{n+1}\right) \mathrm{d} x=\int_{x_{i}}^{x_{i+1 / 2}} W^{i+1 / 2}\left(x, t^{n}\right) \mathrm{d} x-\int_{t^{n}}^{t^{n+1}}\left\langle\mathcal{A}\left(W^{i+1 / 2}(\cdot, t)\right) W_{x}^{i+1 / 2}(\cdot, t), 1_{\left[x_{i}, x_{i+1 / 2}\right]}\right\rangle \mathrm{d} t .
$$

Using (ii) in Proposition 3.1, the above expression can be written as follows:

$$
\int_{x_{i}}^{x_{i+1 / 2}} W^{i+1 / 2}\left(x, t^{n+1}\right) \mathrm{d} x=\int_{x_{i}}^{x_{i+1 / 2}} W^{i+1 / 2}\left(x, t^{n}\right) \mathrm{d} x-\Delta t \int_{0}^{1} \mathcal{A}\left(\Phi\left(s ; W_{i}^{n}, W_{i+1 / 2}^{n}\right)\right) \frac{\partial \Phi}{\partial s}\left(s ; W_{i}^{n}, W_{i+1 / 2}^{n}\right) \mathrm{d} s
$$

where $W_{i+1 / 2}^{n}$ is the constant value of $W^{i+1 / 2}$ at $x=x_{i+1 / 2}$. 
Reasoning as above, we have:

$$
\int_{x_{i-1 / 2}}^{x_{i}} W^{i-1 / 2}\left(x, t^{n+1}\right) \mathrm{d} x=\int_{x_{i-1 / 2}}^{x_{i}} W^{i-1 / 2}\left(x, t^{n}\right) \mathrm{d} x-\Delta t \int_{0}^{1} \mathcal{A}\left(\Phi\left(s ; W_{i-1 / 2}^{n}, W_{i}^{n}\right)\right) \frac{\partial \Phi}{\partial s}\left(s ; W_{i-1 / 2}^{n}, W_{i}^{n}\right) \mathrm{d} s .
$$

Finally, taking into account the initial conditions, we deduce the following expression for the Godunov method:

$$
\begin{aligned}
W_{i}^{n+1}=W_{i}^{n}-\frac{\Delta t}{\Delta x}\left(\int_{0}^{1} \mathcal{A}\left(\Phi\left(s, W_{i-1 / 2}^{n}, W_{i}^{n}\right)\right) \frac{\partial \Phi}{\partial s}(s ;\right. & \left.W_{i-1 / 2}^{n}, W_{i}^{n}\right) \mathrm{d} s \\
& \left.+\int_{0}^{1} \mathcal{A}\left(\Phi\left(s ; W_{i}^{n}, W_{i+1 / 2}^{n}\right)\right) \frac{\partial \Phi}{\partial s}\left(s ; W_{i}^{n}, W_{i+1 / 2}^{n}\right) \mathrm{d} s\right) .
\end{aligned}
$$

Notice that, if the problem is conservative, i.e. if $\mathcal{A}$ is the Jacobian of a flux function $F$, (4.3) gives the usual expression of Godunov method for a system of conservation laws:

$$
W_{i}^{n+1}=W_{i}^{n}-\frac{\Delta t}{\Delta x}\left(F\left(W_{i+1 / 2}^{n}\right)-F\left(W_{i-1 / 2}^{n}\right)\right) .
$$

Remark 4.1. Notice that $W^{i+1 / 2}$ can be discontinuous at $x=x_{i+1 / 2}$. In this case, the discontinuity at $x=x_{i+1 / 2}$ has to be stationary (as the solution is self-similar) and therefore the total mass associated to the corresponding jump is zero (see $(2.3)$ ). Therefore, in the expression of the numerical scheme $W_{i+1 / 2}^{n}$ can be replaced either by the limit of $W^{i+1 / 2}$ to the left, $W_{i+1 / 2}^{n,-}$, or to the right of $x_{i+1 / 2}, W_{i+1 / 2}^{n,+}$. In the particular case of a system of balance laws (3.6) the numerical scheme can be written as follows:

$$
W_{i}^{n+1}=W_{i}^{n}-\frac{\Delta t}{\Delta x}\left(F\left(W_{i+1 / 2}^{n,-}\right)-F\left(W_{i-1 / 2}^{n,+}\right)\right)
$$

Let us investigate now the well-balance property of method (4.3). Well-balancing is related to the numerical approximation of equilibria, i.e., steady state solutions. System (2.1) can only have nontrivial steady state solutions if it has linearly degenerate fields: if $W(x)$ is a regular steady state solution it satisfies

$$
\mathcal{A}(W(x)) \cdot W^{\prime}(x)=0, \quad \forall x \in \mathbb{R}
$$

If $W^{\prime}(x) \neq 0$, then 0 is an eigenvalue of $\mathcal{A}(W(x))$ and $W^{\prime}(x)$ is an associated eigenvector. Therefore, this solution can be interpreted as a parametrization of an integral curve of a linearly degenerate characteristic field whose corresponding eigenvalue takes the value 0 through the curve. In order to define the concept of wellbalancing, let us introduce the set $\Gamma$ of all the integral curves $\gamma$ of any linearly degenerate field of $\mathcal{A}(W)$ such that the corresponding eigenvalue vanishes on $\Gamma$. According to [21], given a curve $\gamma \in \Gamma$, a numerical scheme is said to be exactly well-balanced (respectively well-balanced with order $k$ ) for $\gamma$ if it solves exactly (respectively up to the order $O\left(\Delta x^{k}\right)$ ) regular stationary solutions $W$ satisfying $W(x) \in \gamma$ for every $x$. The numerical scheme is said to be exactly well-balanced (or well-balanced with order $k$ ) if these properties are satisfied for any curve of $\Gamma$ (see [21] for details).

Proposition 4.2. Godunov method (4.3) is exactly well-balanced.

Proof. Let $W$ be a regular stationary solution and let us apply (4.3) to the initial conditions

$$
W_{i}^{0}=W_{0}\left(x_{i}\right), \quad \forall i .
$$


For every $i, W_{i}^{0}$ and $W_{i+1}^{0}$ belong to the same integral curve of a linearly degenerate field whose corresponding eigenvalue takes the value 0 through the curve. As a consequence, the solution of problem (4.2) is a stationary contact discontinuity. Using (4.3) we deduce that, for every $n$,

$$
W_{i}^{n}=W\left(x_{i}\right), \quad \forall i
$$

\section{Consistency of Godunov method for weak solutions}

In [20] a Lax-Wendroff type result was conjectured for path-conservative numerical schemes: if the approximations provided by a path-conservative scheme converge to some function as the mesh is refined, then this function should be a weak solution of the nonconservative system if both the definitions of path-conservative scheme and weak solution make reference to the same family of paths. In this section, we prove a result pointing in that direction for Godunov methods.

We assume that the mesh ratio $\Delta t / \Delta x$ is a fixed constant $\lambda$ as $\Delta x, \Delta t$ tend to 0 and a CFL condition $1 / 2$.

For the discretization of the initial condition we choose the cell averages

$$
W_{i}^{0}=\frac{1}{\Delta x} \int_{x_{i-1 / 2}}^{x_{i+1 / 2}} W_{0}(x) \mathrm{d} x
$$

Set $h=\Delta x$ and introduce the function $W_{h}(x, t)$ defined a.e. in $\mathbb{R} \times[0, \infty)$ by

$$
W_{h}(x, 0)=W_{i}^{0}, \quad x \in\left(x_{i-1 / 2}, x_{i+1 / 2}\right),
$$

and

being $W^{i+1 / 2}$ the solution of (4.2).

$$
W_{h}(x, t)=W^{i+1 / 2}(x, t), \quad(x, t) \in\left(x_{i}, x_{i+1}\right) \times\left[t^{n}, t^{n+1}\right),
$$

The CFL restriction ensures that the adjacent Riemann problems solutions do not interact before time $t^{n+1}$.

Theorem 5.1. For $h>0$, let $W_{h}$ be the numerical approximation (5.2) obtained from the Godunov numerical scheme and $W_{h}(x, 0)$ be given by (5.1).

Suppose that there exists a function $W \in\left(L^{\infty}(\mathbb{R} \times[0, \infty)) \cap B V(\mathbb{R} \times[0, \infty))\right)^{N}$ such that

$$
\left\|W_{h}(\cdot, t)-W(\cdot, t)\right\|_{L^{\infty}(\mathbb{R})^{N}} \underset{h \rightarrow 0}{\longrightarrow} 0 \quad \text { in } L^{1}([0, \infty))
$$

and that there is a constant $C$ such that

$$
T V\left(W_{h}(\cdot, t)\right) \leq C \quad \forall t \in[0, \infty), h>0 .
$$

Then $W$ is a weak solution of the problem (2.1) with initial condition (4.1).

Proof. We want to prove that

$$
\begin{aligned}
\int_{0}^{\infty} \int_{-\infty}^{\infty} W(x, t) \frac{\partial \varphi}{\partial t}(x, t) \mathrm{d} x \mathrm{~d} t-\int_{0}^{\infty}\left\langle\mathcal{A}(W(\cdot, t)) \frac{\partial W}{\partial x}(\cdot, t), \varphi(\cdot, t)\right\rangle \mathrm{d} t & \\
& =-\int_{-\infty}^{+\infty} W_{0}(x) \varphi(x, 0) \mathrm{d} x \quad \forall \varphi \in \mathcal{C}_{0}^{1}(\mathbb{R} \times[0, \infty)) .
\end{aligned}
$$

Let $\varphi \in \mathcal{C}_{0}^{1}(\mathbb{R} \times[0, \infty))$ be a test function and set

$$
\varphi_{i}^{n}=\varphi\left(x_{i}, t^{n}\right) .
$$


By multiplying equation (4.3) by $\varphi_{i}^{n}$ and summing over $i$ and $n$ we have:

$$
\begin{aligned}
\sum_{n=0}^{\infty} \sum_{i=-\infty}^{\infty}\left(W_{i}^{n+1}-W_{i}^{n}\right) \varphi_{i}^{n}+\frac{\Delta t}{\Delta x} \sum_{n=0}^{\infty} \sum_{i=-\infty}^{\infty}\left(\int_{0}^{1} \mathcal{A}\left(\Phi\left(s, W_{i-1 / 2}^{n}, W_{i}^{n}\right)\right) \frac{\partial \Phi}{\partial s}\left(s ; W_{i-1 / 2}^{n}, W_{i}^{n}\right) \mathrm{d} s\right. \\
\left.+\int_{0}^{1} \mathcal{A}\left(\Phi\left(s ; W_{i}^{n}, W_{i+1 / 2}^{n}\right)\right) \frac{\partial \Phi}{\partial s}\left(s ; W_{i}^{n}, W_{i+1 / 2}^{n}\right) \mathrm{d} s\right) \varphi_{i}^{n}=0 .
\end{aligned}
$$

Notice that all these sums are finite since $\varphi$ has compact support.

Applying summation by parts we obtain:

$$
\begin{aligned}
\sum_{n=1}^{\infty} \sum_{i=-\infty}^{\infty} W_{i}^{n}\left(\varphi_{i}^{n}-\varphi_{i}^{n-1}\right)-\frac{\Delta t}{\Delta x} \sum_{n=0}^{\infty} \sum_{i=-\infty}^{\infty} & \left(\int_{0}^{1} \mathcal{A}\left(\Phi\left(s, W_{i+1 / 2}^{n}, W_{i+1}^{n}\right)\right) \frac{\partial \Phi}{\partial s}\left(s ; W_{i+1 / 2}^{n}, W_{i+1}^{n}\right) \mathrm{d} s \varphi_{i+1}^{n}\right. \\
& \left.+\int_{0}^{1} \mathcal{A}\left(\Phi\left(s ; W_{i}^{n}, W_{i+1 / 2}^{n}\right)\right) \frac{\partial \Phi}{\partial s}\left(s ; W_{i}^{n}, W_{i+1 / 2}^{n}\right) \mathrm{d} s \varphi_{i}^{n}\right)=-\sum_{i=-\infty}^{\infty} W_{i}^{0} \varphi_{i}^{0} .
\end{aligned}
$$

Now set

It is straightforward that

$$
\varphi_{i+1 / 2}^{n}=\varphi\left(x_{i+1 / 2}, t^{n}\right)
$$

$$
\varphi_{j}^{n}=\varphi_{i+1 / 2}^{n}+O(\Delta x), \quad j=i, i+1 .
$$

Using this fact and multiplying the last equation by $\Delta x$ we have:

$$
\begin{aligned}
\Delta x \Delta t \sum_{n=1}^{\infty} \sum_{i=-\infty}^{\infty} W_{i}^{n} & \frac{\varphi_{i}^{n}-\varphi_{i}^{n-1}}{\Delta t}-\Delta t \sum_{n=0}^{\infty} \sum_{i=-\infty}^{\infty}\left(\int_{0}^{1} \mathcal{A}\left(\Phi\left(s ; W_{i}^{n}, W_{i+1 / 2}^{n}\right)\right) \frac{\partial \Phi}{\partial s}\left(s ; W_{i}^{n}, W_{i+1 / 2}^{n}\right) \mathrm{d} s\right. \\
& \left.+\int_{0}^{1} \mathcal{A}\left(\Phi\left(s, W_{i+1 / 2}^{n}, W_{i+1}^{n}\right)\right) \frac{\partial \Phi}{\partial s}\left(s ; W_{i+1 / 2}^{n}, W_{i+1}^{n}\right) \mathrm{d} s\right)\left(\varphi_{i+1 / 2}^{n}+O(\Delta x)\right)=-\Delta x \sum_{i=-\infty}^{\infty} W_{i}^{0} \varphi_{i}^{0} .
\end{aligned}
$$

And using (iii) in Proposition 3.1, the last equation can be rewritten as

$$
\begin{array}{r}
\Delta x \Delta t \sum_{n=1}^{\infty} \sum_{i=-\infty}^{\infty} W_{i}^{n} \frac{\varphi_{i}^{n}-\varphi_{i}^{n-1}}{\Delta t}-\Delta t \sum_{n=0}^{\infty} \sum_{i=-\infty}^{\infty}\left(\int_{0}^{1} \mathcal{A}\left(\Phi\left(s ; W_{i}^{n}, W_{i+1}^{n}\right)\right) \frac{\partial \Phi}{\partial s}\left(s ; W_{i}^{n}, W_{i+1}^{n}\right) \mathrm{d} s\right)\left(\varphi_{i+1 / 2}^{n}+O(\Delta x)\right) \\
=-\Delta x \sum_{i=-\infty}^{\infty} W_{i}^{0} \varphi_{i}^{0} .
\end{array}
$$

We want to prove that equation (5.5) can be obtained by passing to the limit as $h$ tends to 0 in (5.6).

It is not difficult to check that the first and the last terms in (5.6) converge to the first and the last terms in (5.5), respectively. It remains to prove that the second term in (5.6) converges to the second one in (5.5).

Because of the smoothness of $\mathcal{A}$ and $\Phi$, and the boundedness of $W_{i}^{n}$ in $L^{\infty}$, it is enough to study the convergence of

$$
\Delta t \sum_{n=0}^{\infty} \sum_{i=-\infty}^{\infty}\left(\int_{0}^{1} \mathcal{A}\left(\Phi\left(s ; W_{i}^{n}, W_{i+1}^{n}\right)\right) \frac{\partial \Phi}{\partial s}\left(s ; W_{i}^{n}, W_{i+1}^{n}\right) \mathrm{d} s\right) \varphi_{i+1 / 2}^{n} .
$$

Using Proposition 3.1 and the CFL condition, the following equality holds for every $t \in\left[t^{n}, t^{n+1}\right)$,

$$
\left\langle\mathcal{A}\left(W_{h}(\cdot, t)\right) \frac{\partial W_{h}}{\partial x}(\cdot, t), 1_{\left[x_{i}, x_{i+1}\right]}\right\rangle=\int_{0}^{1} \mathcal{A}\left(\Phi\left(s ; W_{i}^{n}, W_{i+1}^{n}\right)\right) \frac{\partial \Phi}{\partial s}\left(s ; W_{i}^{n}, W_{i+1}^{n}\right) \mathrm{d} s .
$$


From this equality, we find that

$$
\begin{array}{r}
\Delta t \sum_{n=0}^{\infty} \sum_{i=-\infty}^{\infty}\left(\int_{0}^{1} \mathcal{A}\left(\Phi\left(s ; W_{i}^{n}, W_{i+1}^{n}\right)\right) \frac{\partial \Phi}{\partial s}\left(s ; W_{i}^{n}, W_{i+1}^{n}\right) \mathrm{d} s\right) \varphi_{i+1 / 2}^{n}=\int_{0}^{\infty}\left\langle\mathcal{A}\left(W_{h}(\cdot, t)\right) \frac{\partial W_{h}}{\partial x}(\cdot, t), \varphi(\cdot, t)\right\rangle \mathrm{d} t \\
+\sum_{n=0}^{\infty} \sum_{i=-\infty}^{\infty} \int_{t^{n}}^{t^{n+1}}\left\langle\mathcal{A}\left(W_{h}(\cdot, t)\right) \frac{\partial W_{h}}{\partial x}(\cdot, t),\left(\varphi_{i+1 / 2}^{n}-\varphi(\cdot, t)\right) 1_{\left[x_{i}, x_{i+1}\right]}\right\rangle \mathrm{d} t
\end{array}
$$

The second summand trivially converges to 0 . To study the convergence of the first summand we use the following convergence result shown in [5]:

Theorem 5.2. Let $U_{k}$ be a sequence in $(B V(a, b))^{N}$ and $U$ be in $(B V(a, b))^{N}$ such that

$$
U_{k} \underset{k \rightarrow \infty}{\longrightarrow} U \text { in }\left(L^{\infty}(a, b)\right)^{N}
$$

and that there exists a constant $C$ such that

$$
T V_{a}^{b}\left(U_{k}\right) \leq C \quad \forall k
$$

Then, for every continuous function $a: \mathbb{R}^{N} \rightarrow \mathbb{R}^{N}$, we have:

$$
\left[a\left(U_{k}\right) \frac{\mathrm{d} U_{k}}{\mathrm{~d} x}\right]_{\Phi} \underset{k \rightarrow \infty}{\longrightarrow}\left[a(U) \frac{\mathrm{d} U}{\mathrm{~d} x}\right]_{\Phi}
$$

weakly-star in the sense of bounded measures, i.e.

$$
\left\langle\left[a\left(U_{k}\right) \frac{\mathrm{d} U_{k}}{\mathrm{~d} x}\right]_{\Phi}, \varphi\right\rangle \underset{k \rightarrow \infty}{\longrightarrow}\left\langle\left[a(U) \frac{\mathrm{d} U}{\mathrm{~d} x}\right]_{\Phi}, \varphi\right\rangle \quad \forall \varphi \in \mathcal{C}([a, b]) .
$$

Using this result we find that

$$
\left\langle\mathcal{A}\left(W_{h}(\cdot, t)\right) \frac{\partial W_{h}}{\partial x}(\cdot, t), \varphi(\cdot, t)\right\rangle \underset{h \rightarrow 0}{\longrightarrow}\left\langle\mathcal{A}(W(\cdot, t)) \frac{\partial W}{\partial x}(\cdot, t), \varphi(\cdot, t)\right\rangle .
$$

The Lebesgue dominated convergence theorem allows to prove the convergence of the first summand in (5.7) to

$$
\int_{0}^{\infty}\left\langle\mathcal{A}(W(\cdot, t)) \frac{\partial W}{\partial x}(\cdot, t), \varphi(\cdot, t)\right\rangle \mathrm{d} t
$$

as $h$ tends to 0 , which concludes the proof.

Remark 5.3. Theorem 5.1 cannot be considered as an extension of the classical Lax-Wendroff theorem for Godunov methods in the context of nonconservative problems, as the uniform convergence required here is much stronger than that required in the classical result. Moreover, the hypothesis concerning the total variation is not realistic: in [2] it has been shown that Godunov methods can increase arbitrarily the total variation even in the context of conservative problems. The negative results shown in [13] or [6] concerning the failure of the convergence of nonconservative schemes must also be taken into account in further investigations. Therefore, the previous result has to be understood merely as a consistency result of the numerical scheme with the chosen notion of weak solution and, thus, with the corresponding Rankine-Hugoniot conditions. Some results of convergence for Godunov methods applied to systems of balance laws can be found in [14] or [9]. 
Remark 5.4. Theorem 5.1 does not imply that the weak solution obtained as the mesh is refined is an entropy solution. If the definition of entropy solution is related to an entropy pair (see Def. 2.4) we should prove that

$$
\begin{aligned}
\int_{0}^{\infty} \int_{-\infty}^{\infty} \eta(W(x, t)) \frac{\partial \varphi}{\partial t}(x, t) \mathrm{d} x \mathrm{~d} t-\int_{0}^{\infty} \int_{-\infty}^{\infty} & G(W(x, t)) \frac{\partial \varphi}{\partial x}(x, t) \mathrm{d} x \mathrm{~d} t \\
& \leq-\int_{-\infty}^{+\infty} \eta\left(W_{0}(x)\right) \varphi(x, 0) \mathrm{d} x \quad \forall \varphi \in \mathcal{C}_{0}^{1}\left(\mathbb{R} \times \mathbb{R}^{+}\right), \varphi \geq 0
\end{aligned}
$$

To show this, it is enough to have a discrete entropy inequality of the form

$$
\eta\left(W_{i}^{n+1}\right) \leq \eta\left(W_{i}^{n}\right)-\frac{\Delta t}{\Delta x}\left(\mathcal{G}_{i+1 / 2}^{n}-\mathcal{G}_{i-1 / 2}^{n}\right)
$$

where

$$
\mathcal{G}_{i+1 / 2}^{n}=\mathcal{G}\left(W_{i-q}^{n}, \ldots, W_{i+p}^{n}\right),
$$

$\mathcal{G}$ being a Lipschitz continuous function from $\Omega^{p+q+1}$ to $\Omega$ consistent with $G$ in the sense that

$$
\mathcal{G}(W, \ldots, W)=G(W) \quad \forall W \in \Omega
$$

The proof is identical to that corresponding to conservative schemes (see [15]).

If $\eta$ is a convex function and $\left(W_{L}, W_{R}\right) \in \mathcal{R} \mathcal{P}$, the following inequality can be proved for the solution of a Riemann problem:

$$
\begin{aligned}
& \mathcal{G}_{R}\left(W_{L}, W_{R}\right):=G\left(W_{R}\right)+\int_{0}^{\infty}\left(\eta\left(V\left(v ; W_{L}, W_{R}\right)\right)-\eta\left(W_{R}\right)\right) \mathrm{d} v \\
& \quad \leq \mathcal{G}_{L}\left(W_{L}, W_{R}\right):=G\left(W_{L}\right)-\int_{-\infty}^{0}\left(\eta\left(V\left(v ; W_{L}, W_{R}\right)\right)-\eta\left(W_{L}\right)\right) \mathrm{d} v
\end{aligned}
$$

being $V\left(x / t ; W_{L}, W_{R}\right)$ the self-similar solution of the Riemann problem (3.1). Again, the proof is identical to that corresponding to systems of conservation laws (see [1]). It can be easily shown that (5.13) is satisfied for any numerical entropy flux function $\mathcal{G}\left(W_{L}, W_{R}\right)$ such that:

$$
\mathcal{G}_{R}\left(W_{L}, W_{R}\right) \leq \mathcal{G}\left(W_{L}, W_{R}\right) \leq \mathcal{G}_{L}\left(W_{L}, W_{R}\right)
$$

\section{Path-Approximate Riemann solvers}

In [20] the concept of path-conservative numerical scheme was introduced:

Definition 6.1. Given a family of paths $\Psi$, a numerical scheme is said to be $\Psi$-conservative if it can be written in the form

$$
W_{i}^{n+1}=W_{i}^{n}-\frac{\Delta t}{\Delta x}\left(D_{i-1 / 2}^{+}+D_{i+1 / 2}^{-}\right)
$$

where

$$
D_{i+1 / 2}^{ \pm}=D^{ \pm}\left(W_{i-q}^{n}, \ldots, W_{i+p}^{n}\right)
$$

$D^{-}$and $D^{+}$being two continuous functions from $\Omega^{p+q+1}$ to $\Omega$ satisfying:

$$
D^{ \pm}(W, \ldots, W)=0, \quad \forall W \in \Omega
$$


and

$$
D^{-}\left(W_{-q}, \ldots, W_{p}\right)+D^{+}\left(W_{-q}, \ldots, W_{p}\right)=\int_{0}^{1} \mathcal{A}\left(\Psi\left(s ; W_{0}, W_{1}\right)\right) \frac{\partial \Psi}{\partial s}\left(s ; W_{0}, W_{1}\right) \mathrm{d} s
$$

for every $W_{i} \in \Omega, i=-q, \ldots, p$.

The notion of approximate Riemann solver was introduced in [12] for conservative systems and extended for balance laws in [1]. In [20] the following generalization for nonconservative systems (2.1) was introduced:

Definition 6.2. Given a family of paths $\Psi$, a $\Psi$-approximate Riemann solver for (2.1) is a function $\widetilde{V}$ : $\mathbb{R} \times \Omega \times \Omega \mapsto \Omega$ satisfying:

- For every $W \in \Omega$ :

$$
\widetilde{V}(v ; W, W)=W, \quad \forall v \in \mathbb{R} .
$$

- For every $W_{L}, W_{R} \in \Omega$ there exist $\lambda_{\min }\left(W_{L}, W_{R}\right), \lambda_{\max }\left(W_{L}, W_{R}\right)$ in $\mathbb{R}$ such that:

$$
\begin{aligned}
& \tilde{V}\left(v ; W_{L}, W_{R}\right)=W_{L}, \quad \text { if } v<\lambda_{\min }\left(W_{L}, W_{R}\right) \\
& \tilde{V}\left(v ; W_{L}, W_{R}\right)=W_{R}, \quad \text { if } v>\lambda_{\max }\left(W_{L}, W_{R}\right) .
\end{aligned}
$$

- For every $W_{L}, W_{R} \in \Omega$ :

$$
\begin{aligned}
\int_{0}^{1} \mathcal{A}\left(\Psi\left(s ; W_{L}, W_{R}\right)\right) \frac{\partial \Psi}{\partial s}\left(s ; W_{L}, W_{R}\right) \mathrm{d} s & \\
& +\int_{0}^{\infty}\left(\tilde{V}\left(v ; W_{L}, W_{R}\right)-W_{R}\right) \mathrm{d} v+\int_{-\infty}^{0}\left(\tilde{V}\left(v ; W_{L}, W_{R}\right)-W_{L}\right) \mathrm{d} v=0 .
\end{aligned}
$$

Given a $\Psi$-approximate Riemann solver for (2.1) a numerical scheme can be constructed as follows:

$$
W_{i}^{n+1}=\frac{1}{\Delta x}\left(\int_{x_{i-1 / 2}}^{x_{i}} \tilde{V}\left(\frac{x-x_{i-1 / 2}}{\Delta t} ; W_{i-1}^{n}, W_{i}^{n}\right) \mathrm{d} x+\int_{x_{i}}^{x_{i+1 / 2}} \tilde{V}\left(\frac{x-x_{i+1 / 2}}{\Delta t} ; W_{i}^{n}, W_{i+1}^{n}\right) \mathrm{d} x\right)
$$

Under a CFL condition $1 / 2$, the numerical scheme can also be written in the form (6.1) with

$$
\begin{aligned}
& D_{i+1 / 2}^{-}=-\int_{-\infty}^{0}\left(\widetilde{V}\left(v ; W_{i}^{n}, W_{i+1}^{n}\right)-W_{i}^{n}\right) \mathrm{d} v \\
& D_{i+1 / 2}^{+}=-\int_{0}^{\infty}\left(\widetilde{V}\left(v ; W_{i}^{n}, W_{i+1}^{n}\right)-W_{i+1}^{n}\right) \mathrm{d} v .
\end{aligned}
$$

Clearly, a numerical scheme (6.1) based on a $\Psi$-approximate Riemann solver is $\Psi$-conservative.

Godunov method is the particular case corresponding to the choice of the exact Riemann solver, i.e.

$$
\widetilde{V}\left(v ; W_{L}, W_{R}\right)=V\left(v ; W_{L}, W_{R}\right)
$$

Let us consider a path-numerical scheme based on a $\Phi$-approximate Riemann solver, $\Phi$ being the family of paths used for the definition of weak solutions. We consider again a function $W_{h}(x, t)$ defined as in the previous section, just replacing (5.2) by:

$$
W_{h}(x, t)=\widetilde{V}\left(\frac{x-x_{i+1 / 2}}{t-t^{n}} ; W_{i}^{n}, W_{i+1}^{n}\right), \quad(x, t) \in\left(x_{i}, x_{i+1}\right) \times\left[t^{n}, t^{n+1}\right) .
$$


Theorem 5.1 remains valid for $W_{h}$ provided that the approximate Riemann solver satisfies the equality:

$$
\left\langle\mathcal{A}\left(\widetilde{V}\left(x / t ; W_{L}, W_{R}\right)\right) \frac{\partial}{\partial x} \widetilde{V}\left(x / t ; W_{L}, W_{R}\right), 1_{[A, B]}(x)\right\rangle=\int_{0}^{1} \mathcal{A}\left(\Phi\left(s ; W_{L}, W_{R}\right)\right) \frac{\partial \Phi}{\partial s}\left(s ; W_{L}, W_{R}\right) \mathrm{d} s,
$$

for every $W_{L}, W_{R}, t \geq 0$, and $A, B$ such that:

$$
A \leq \lambda_{\min }\left(W_{L}, W_{R}\right) t, \quad \lambda_{\max }\left(W_{L}, W_{R}\right) t \leq B .
$$

The proof is similar to that of Theorem 5.1.

Moreover, if the concept of entropy is given by an entropy pair with convex $\eta$ and the $\Psi$-approximate Riemann solver is assumed to be dissipative in the sense that the following inequality is satisfied for every $W_{L}, W_{R}$ :

$$
\begin{aligned}
\widetilde{\mathcal{G}}_{R}\left(W_{L}, W_{R}\right):=G\left(W_{R}\right)+\int_{0}^{\infty}\left(\eta\left(\widetilde{V}\left(v ; W_{L}, W_{R}\right)\right)-\eta\left(W_{R}\right)\right) \mathrm{d} v & \\
& \quad \leq \widetilde{\mathcal{G}}_{L}\left(W_{L}, W_{R}\right):=G\left(W_{L}\right)-\int_{-\infty}^{0}\left(\eta\left(\widetilde{V}\left(v ; W_{L}, W_{R}\right)\right)-\eta\left(W_{L}\right)\right) \mathrm{d} v,
\end{aligned}
$$

then (5.12) is satisfied for any numerical entropy flux function $\mathcal{G}\left(W_{L}, W_{R}\right)$ satisfying

$$
\widetilde{\mathcal{G}}_{R}\left(W_{L}, W_{R}\right) \leq \mathcal{G}\left(W_{L}, W_{R}\right) \leq \widetilde{\mathcal{G}}_{L}\left(W_{L}, W_{R}\right)
$$

In this case, the weak solution obtained as the mesh is refined is an entropy solution.

Acknowledgements. This research has been partially supported by the Spanish Government Research projects BFM200307530-C02-02 and MTM2006-08075.

\section{REFERENCES}

[1] F. Bouchut, Nonlinear Stability of Finite Volume Methods for Hyperbolic Conservation Laws and Well-Balanced Schemes for Sources. Birkhäuser (2004).

[2] A. Bressan, H.K. Jenssen and P. Baiti, An instability of the Godunov Scheme. arXiv:math.AP/0502125 v2 (2005).

[3] M.J. Castro, J. Macías and C. Parés, A Q-Scheme for a class of systems of coupled conservation laws with source term. Application to a two-layer 1-D shallow water system. Math. Mod. Num. Anal. 35 (2001) 107-127.

[4] F. Coquel, D. Diehl, C. Merkle and C. Rohde, Sharp and diffuse interface methods for phase transition problems in liquidvapour flows, in Numerical Methods for Hyperbolic and Kinetic Problems, IRMA Lectures in Mathematics and Theoretical Physics, Proceedings of CEMRACS 2003.

[5] G. Dal Maso, P.G. LeFloch and F. Murat, Definition and weak stability of nonconservative products. J. Math. Pures Appl. 74 (1995) 483-548.

[6] F. De Vuyst, Schémas non-conservatifs et schémas cinétiques pour la simulation numérique d'écoulements hypersoniques non visqueux en déséquilibre thermochimique. Ph.D. thesis, University of Paris VI, France (1994).

[7] E. Godlewski and P.A. Raviart, Numerical Approximation of Hyperbolic Systems of Conservation Laws. Springer (1996).

[8] L. Gosse, A well-balanced flux-vector splitting scheme designed for hyperbolic systems of conservation laws with source terms. Comput. Math. Appl. 39 (2000) 135-159.

[9] L. Gosse, A well-balanced scheme using non-conservative products designed for hyperbolic systems of conservation laws with source terms. Math. Models Methods Appl. Sci. 11 (2001) 339-365.

[10] J.M. Greenberg and A.Y. LeRoux, A well balanced scheme for the numerical processing of source terms in hyperbolic equations. SIAM J. Numer. Anal. 33 (1996) 1-16.

[11] J.M. Greenberg, A.Y. LeRoux, R. Baraille and A. Noussair, Analysis and approximation of conservation laws with source terms. SIAM J. Numer. Anal. 34 (1997) 1980-2007.

[12] A. Harten, P.D. Lax and B. Van Leer, On upstream differencing and Godunov-type schemes for hyperbolic conservation laws. SIAM Rev. 25 (1983) 35-61.

[13] T. Hou and P.G. LeFloch, Why nonconservative schemes converge to wrong solutions: error analysis. Math. Comp. 62 (1994) $497-530$. 
[14] E. Isaacson and B. Temple, Convergence of the $2 \times 2$ Godunov method for a general resonant nonlinear balance law. SIAM J. Appl. Math. 55 (1995) 625-640.

[15] P.D. Lax and B. Wendroff, Systems of conservation laws. Comm. Pure Appl. Math. 13 (1960) 217-237.

[16] P.G. LeFloch, Shock waves for nonlinear hyperbolic systems in nonconservative form. Institute Math. Appl., Minneapolis, Preprint 593 (1989).

[17] P.G. LeFloch, Graph solutions of nonlinear hyperbolic systems. J. Hyper. Differ. Equa. 2 (2004) 643-689.

[18] P.G. LeFloch and A.E. Tzavaras, Representation of weak limits and definition of nonconservative products. SIAM J. Math. Anal. 30 (1999) 1309-1342.

[19] R.J. LeVeque, Balancing source terms and flux gradients in high-resolution Godunov methods: the quasi-steady wavepropagation algorithm. J. Comp. Phys. 146 (1998) 346-365.

[20] C. Parés, Numerical methods for nonconservative hyperbolic systems: a theoretical framework. SIAM J. Numer. Anal. 44 (2006) 300-321.

[21] C. Parés and M. Castro, On the well-balance property of Roe's method for nonconservative hyperbolic systems. Applications to shallow water systems. Math. Mod. Num. Anal. 38 (2004) 821-852.

[22] A.I. Volpert, The space BV and quasilinear equations. Math. USSR Sbornik 73 (1967) 225-267. 